\title{
Spin-Orbit Coupling Effect on the Electrophilicity Index, Chemical Potential, Hardness and Softness of Neutral Gold Clusters: A Relativistic Ab-initio Study
}

\author{
Mahnaz Jabbarzadeh Sani ${ }^{a^{*}}$ \\ ${ }^{a}$ Department of Chemistry, College of Science, Shiraz University, Shiraz, Iran.
}

\begin{abstract}
The Electrophilicity index $(\omega)$ is related to the energy lowering associated with the maximum amount of electron flow between a donor and an acceptor and possesses adequate information regarding structure, stability, reactivity, and interactions. Chemical potential $(\mu)$ measures charge transfer from a system to another having a lower value of $\mu$, while chemical hardness $(\eta)$ is a measure of the characterized relative stability of clusters. The main purpose of the present research work is to examine the Spin-Orbit Coupling (SOC) effect on the behavior of the electrophilicity index, chemical potential, hardness and softness of neutral gold clusters $\mathrm{Au}_{n}(\mathrm{n}=2-6)$. Using the second-order Douglas-Kroll-Hess Hamiltonian, geometries are optimized at the DKH2-B3P86/DZP-DKH level of theory. Spin-orbit coupling energies are computed using the fourth-order Douglas-Kroll-Hess Hamiltonian, generalized Hartree-Fock method and all-electron relativistic double- $\zeta$ level basis set. Then, spin-orbit coupling (SOC) corrections to the electrophilicity index, chemical potential, hardness and softness are calculated. It is revealed that spin-orbit correction to the vertical chemical hardness has an important effect on $\mathrm{Au}_{3}$ and $\mathrm{Au}_{6}$, i.e. SOC decreases (increases) the hardness of gold trimer (hexamer). Due to the relationship between hardness and softness, $\sigma=1 / \eta$, inclusion of spin-orbit coupling increases (decreases) the softness of $\mathrm{Au}_{3}\left(\mathrm{Au}_{6}\right)$ and thus destabilizes (stabilizes) it. Spin-orbit coupling (SOC) also has an important effect on the chemical potential of $\mathrm{Au}_{3}$ by decreasing its value. It is found that spin-orbit coupling has a considerable effect on the electrophilicity index of gold trimer and greatly increases its value. Furthermore, SOC increases the maximal charge acceptance of $\mathrm{Au}_{3}$ more and thus destabilizes it more. As a result, the spin-orbit coupling effect appears to be important in calculating the electrophilicity index of the gold trimer.
\end{abstract}

Keywords: Density Functional Theory; Douglas-Kroll-Hess; Electronic Properties; Gold Clusters; Spin-orbit Coupling.

\section{Introduction}

As a kind of promising nanomaterial, metal nanoclusters (NCs) have sparked wide-spread attention. In recent years, gold nanoparticles (AuNPs) have been applied to biomedicine and biological sensing [1-5] due to their biocompatibility and unique physical properties. AuNPs are one of the most promising catalysts, in spite of bulk Au as an inactive material [6-8]. Most of the computations on small neutral gold clusters have been performed using spinfree (scalar-relativistic) methods [9-12]. To obtain reliable theoretical results for gold clusters, the scalar relativistic correction is substantial. However, the spin-orbit coupling is expected to be important. There exist theoretical studies regarding spin-orbit coupling effects using effective core potentials or plane-wave basis sets [13-19]. These studies mainly focused on the spin-orbit coupling effect on the highest-occupied, lowest-unoccupied (HOMO-LUMO) energy gaps, geometries, binding energies per atom, and optical absorption of gold clusters. Xiao and Wang [13] using

* Corresponding author: mjsani@shirazu.ac.ir

$>$ This is an open access article under the CC-BY license (https://creativecommons.org/licenses/by/4.0/).

(C) Authors retain all copyrights. 
PW91PW91 functional and a plane-wave basis set concluded that the spin-orbit coupling increases the binding energy for all the clusters, Aun $(n=5-11,14,20)$, within the same magnitude. They also found that spin-orbit coupling decreases the HOMO-LUMO energy gap, but has no effect on the magnetic moment. Using a spin-averaged relativistic effective core potential, Li et al. [14] evaluated the performance of various density functional theory methods on the geometries and atomization energies of $\mathrm{Au}_{2}, \mathrm{Au}_{3}, \mathrm{Au}_{4}$ and $\mathrm{Au}_{5}$. They included spin-orbit (SO) coupling effect using two-component spinor formalism. They found that the functionals, including the SO coupling effect, overestimate the atomization energies of gold clusters compared with those just including the scalar-relativistic (SC) effect. Flores and Proupin [15], applying PBE functional and pseudopotentials, have investigated the effect of the spin-orbit coupling on the structures, relative stability, and HOMO-LUMO energy gap of the lowest-lying isomers of the neutral $\mathrm{Au}_{13}$ cluster. Rusakov et al. [16] have used two-component relativistic density functional theory with accurate small-core shape-consistent relativistic pseudopotentials and spin-orbit corrections to refine the isomerization energy profile of $\mathrm{Au}_{3}$ computed by spin-orbit free coupled cluster methods. The noble metal trimers, $\mathrm{Au}_{3}, \mathrm{Ag}_{3}$ and $\mathrm{Cu}_{3}$, have also been investigated by applying the four-component Kohn-Sham-Dirac equation in the framework of relativistic density functional theory [17].

The concept of electrophilicity has been known for several decades. Parr et al. [20] proposed a definition based on the energy lowering associated with a maximum amount of electron flow between two species. It has been revealed that electrophilicity possesses adequate information regarding structure, stability, reactivity and interactions [21]. In the present work, spin-orbit coupling (SOC) effects on the electronic properties of small neutral gold clusters, $\mathrm{Au}_{\mathrm{n}}$ $(n=2-6)$, are investigated applying the Douglas-Kroll-Hess Hamiltonian and all-electron relativistic basis set of valence double- $\zeta$ quality plus polarization functions (DZP-DKH). The spin-orbit coupling effects on the electrophilicity index, electronic chemical potential, absolute hardness and softness are examined.

\section{Computational Methods}

All calculations are performed using the Gaussian 09 suite of program [22] and the plots of molecular configurations and contour maps are generated with the GaussView software [23]. The B3P86 functional is used in conjunction with the valence double- $\zeta$ quality plus polarization functions (DZP-DKH [8s7p4d2f]) basis set [24]. The B3P86 functional has already proven to perform well for ionization potential computations of small neutral gold clusters [10]. The second-order Douglas-Kroll-Hess Hamiltonian [25-29] is used instead of the Schrödinger operator. For geometry optimizations, the coordinates are chosen according to the experimentally determined structures by Gruene et al. [30]. Using the second-order Douglas-Kroll-Hess Hamiltonian, all geometries are fully optimized at the DKH2-B3P86/DZP-DKH level of theory followed by harmonic vibrational frequency analysis. Then, from these optimized geometries, the spin-orbit coupling (SOC) energies are calculated using the fourth-order Douglas-KrollHess Hamiltonian and the generalized Hartree-Fock method [31]. The electronic energy including spin-orbit coupling, $E_{S O}$, is calculated using the following definition,

$E_{S O}=E_{S C}+\Delta_{S O} E$

Where, $E_{S C}$ is the electronic energy obtained from spin-orbit free (scalar-relativistic) optimizations at the DKH2B3P86/DZP-DKH level, and $\Delta_{S O} E$ is the spin-orbit coupling energy calculated by the DKH2-B3P86/DZP-DKH //DKHSO- GHF/DZP-DKH level of theory. In order to determine the spin-orbit coupling (SOC) effect on the electronic properties of neutral gold clusters, the spin-orbit corrections to the chemical hardness, softness, chemical potential and electrophilicity index $[20,32]$ of the gold clusters are computed. In the following, the spin-orbit correction to a particular property, $\Delta_{S O} P$, is defined using,

$\Delta_{S O} P=P_{S O}-P_{S C}$

Where, $P_{S O}$ and $P_{S C}$ are the calculated properties with considering the spin-orbit coupling energy, $E_{S O}$, and without considering the spin-orbit coupling energy, $E_{S C}$, respectively. Within the valence state parabola model [20], the chemical potential, chemical hardness, softness and electrophilicity index are introduced by,

$\mu=\left(\frac{\partial E}{\partial N}\right)_{v}=-\frac{I P+E A}{2}$

$\eta=\left(\frac{\partial^{2} E}{\partial N^{2}}\right)_{v}=\frac{I P-E A}{2}$

$\sigma=\frac{1}{\eta}$

$\omega=\frac{\mu^{2}}{2 \eta}$

Where N, v, IP and EA are the number of electrons, the potential due to the nuclei plus any external potential, ionization potential and electron affinity, respectively. The spin-orbit corrections to these properties are calculated from, 
$\Delta_{S O} \mu=\mu_{S O}-\mu_{S C}$

$\Delta_{S O} \eta=\eta_{S O}-\eta_{S C}$

$\Delta_{S O} \sigma=\sigma_{S O}-\sigma_{S C}$

$\Delta_{S O} \omega=\omega_{S O}-\omega_{S C}$

The aforementioned properties are calculated using the both vertical and adiabatic ionization potential and electron affinities. Figure 1 illustrates the steps in calculating the spin-orbit coupling (SOC) effect on the electrophilicity index, chemical potential, hardness and softness of neutral gold clusters.

1. Selection of the appropriate coordinates for the: optimization.

2. Selection of the appropriate method and basis set for the geometry optimization (This work: DKH2-B3P86/DZP-DKH level).

3. Selection of the correct Hamiltonian for the relativistic calculations (This work: Douglas-Kroll-Hess (DKH) Hamiltonian).

4. Optimization of the selected geometries at the DKH2-B3P86/DZPDKH level and applying DKH Hamiltonian.

5. Performing a harmonic vibrational frequency analysis to characterize the nature of the stationary points.

6. Calculation of the spin-orbit coupling energies at the DKH2B3P86/DZP-DKH //DKHSO- GHF/DZP-DKH level of theory $\left(\Delta_{S O} E\right.$ $=\langle L . S\rangle=$ spin-orbit coupling energy).

7. Calculation of the electronic energy including spin-orbit coupling:

$$
E_{S O}=E_{S C}+\Delta_{S O} E
$$

8. Calculation of the properties (using the both $E_{S C}$ and $E_{S O}$ ):

- Chemical potential $\mu=-\frac{I P+E A}{2}$

- Hardness $\eta=\frac{I P-E A}{2}$

- Softness $\sigma=\frac{1}{\eta}$

- Electrophilicity index $\omega=\frac{\mu^{2}}{2 \eta}$

9. Calculation of the spin-orbit corrections to these properties:

- $\Delta_{S O} \mu=\mu_{S O}-\mu_{S C}$

- $\Delta_{S O} \eta=\eta_{S O}-\eta_{S C}$

- $\Delta_{S O} \sigma=\sigma_{S O}-\sigma_{S C}$

- $\Delta_{S O} \omega=\omega_{S O}-\omega_{S C}$

Figure 1. Flow diagram showing the computational steps 


\section{Results and Discussion}

The optimized structures of gold clusters at the DKH2-B3P86/DZP-DKH level of theory are displayed in Figure 2. Table 1 compares the apex angle, symmetry and electronic state of the two Jahn-Teller components of gold trimer. The isomer with the lowest energy is an acute angle triangular structure with $\mathrm{C}_{2 \mathrm{~V}}$ symmetry, a $58.455^{\circ}$ apex angle, and a $2.537 \mathrm{~A}^{\circ}$ bond length. The result is in agreement with the coupled cluster calculations of Schwerdtfeger et al. [33]. In fact, because of the Jahn-Teller effect, the $\mathrm{D}_{3 \mathrm{~h}}$ symmetry of $\mathrm{Au}_{3}$ distorts to an acute triangular $\mathrm{C}_{2 \mathrm{~V}}$ structure. The lowest-energy structures of $\mathrm{Au}_{4}$ and $\mathrm{Au}_{5}$ are a trapezoid with $\mathrm{C}_{2} \mathrm{~V}$ symmetry and a W-shaped geometry, respectively. The $\mathrm{D}_{3 \mathrm{~h}}$ planar triangular structure of $\mathrm{Au}_{6}$ is obtained by adding one gold atom to W-shaped planar $\mathrm{Au}_{5}$.

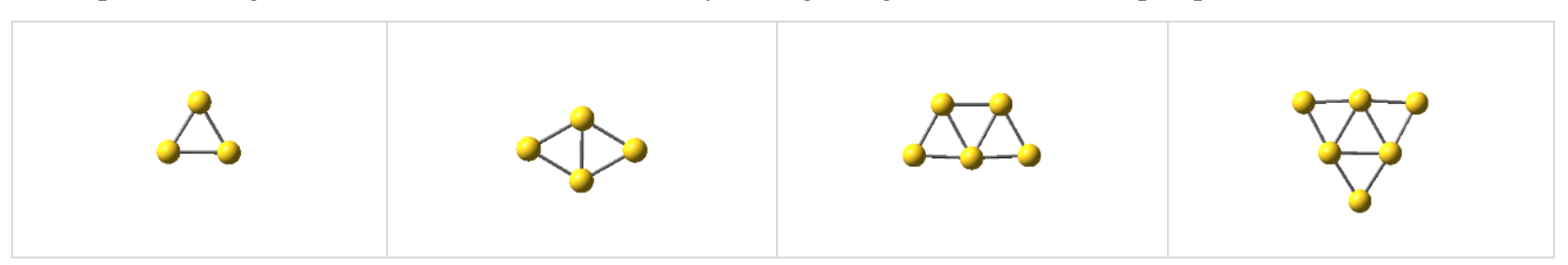

Figure 2. The calculated structures of gold clusters (Aun, $n=3-6$ ) at the DKH2-B3P86/DZP-DKH level of theory

Table 1. Apex angle, symmetry and electronic state of the two Jahn-Teller components of gold trimer

\begin{tabular}{cccc}
\hline & Apex angle & Symmetry & Electronic state \\
\hline $\mathrm{Au}_{3}$ (acute) & $58.455^{\circ}$ & $\mathrm{C}_{2 \mathrm{~V}}$ & ${ }^{2} \mathrm{~A}_{1}$ \\
$\mathrm{Au}_{3}$ (obtuse) & $62.447^{\circ}$ & $\mathrm{C}_{2 \mathrm{~V}}$ & ${ }^{2} \mathrm{~B}_{2}$ \\
\hline
\end{tabular}

The acute gold trimer has lower energy than the obtuse one at the DKH2-B3P86/DZP-DKH level of theory.

The calculated vertical and adiabatic ionization potential, electron affinity, chemical potential, hardness, softness and electrophilicity index values are given in Tables 2 to 5 . Table 6 presents the spin-orbit coupling (SOC) corrections to the chemical potential, hardness, softness and electrophilicity index. Chemical hardness has been used to characterize the relative stability of clusters. The principle of maximum hardness (PMH) states that systems at equilibrium present the highest value of hardness [34]. The computed hardness values with and without considering spin-orbit coupling (SOC) effect are plotted in Figure 3a. According to this Figure, the chemical hardness computed using adiabatic electron affinities and ionization potentials exhibits an odd-even oscillation behavior, whether spinorbit coupling is considered or not. The even-sized clusters with closed-shell electronic configurations have higher values of chemical hardness compared to their immediate open-shell neighbors, indicating their higher stability. This is in agreement with the previous study of Singh and Sarkar [11]. They performed spin-free (scalar- relativistic) calculations using B3LYP/LANL2DZ method. The spin-orbit correction to the chemical hardness with the increasing cluster size is presented in Figure 4a. As this Figure shows, the spin-orbit correction to the vertical chemical hardness has important effect on $\mathrm{Au}_{3}$ and $\mathrm{Au}_{6}$. The $\boldsymbol{\Delta}_{\text {so }} \eta_{v}$ value for acute $\mathrm{Au}_{3}$ and $\mathrm{Au}_{6}$ is negative $(-0.038 \mathrm{eV})$ and positive $(0.045 \mathrm{eV})$, respectively, i.e. spin-orbit coupling decreases (increases) the hardness of gold trimer (hexamer).

The softness, $\sigma$, is simply the inverse of the hardness. Figure $3 b$ depicts the variation of softness as a function of cluster size, with and without considering spin-orbit coupling (SOC) effect. As this Figure illustrates, the oscillation behavior of softness is opposite to that of chemical hardness, due to its relationship with hardness, $\sigma=1 / \eta$. Moreover, the obtuse $\mathrm{Au}_{3}$ has the highest adiabatic softness $\left(\sigma_{\mathrm{a}}=0.454 \mathrm{eV}^{-1}\right.$ and $\left.\sigma_{\mathrm{a}, \mathrm{so}}=0.456 \mathrm{eV}^{-1}\right)$, indicating its high reactivity. On the other hand, hard molecules have a large HOMO-LUMO gap, whereas soft molecules have a small energy gap [32]. The HOMO-LUMO energy gap of $\mathrm{Au}_{2}$, obtuse (acute) $\mathrm{Au}_{3}, \mathrm{Au}_{4}, \mathrm{Au}_{5}$ and $\mathrm{Au}_{6}$ at the DKH2-B3P86/DZP-DKH level of theory is $2.760,1.170(1.454), 1.982,1.529$ and $2.739 \mathrm{eV}$, respectively. Therefore, obtuse $\mathrm{Au}_{3}$ with the highest adiabatic softness has the lowest HOMO-LUMO gap value of $1.170 \mathrm{eV}$. The variation of the spin-orbit correction to the softness of neutral gold clusters as a function of cluster size is plotted in Figure 4b. The spin-orbit correction to the softness shows an even-odd alternation behavior. Furthermore, the inclusion of spin-orbit coupling increases (decreases) the softness of $\mathrm{Au}_{3}\left(\mathrm{Au}_{6}\right)$ and thus destabilizes (stabilizes) it, $\boldsymbol{\Delta}_{\boldsymbol{s} \boldsymbol{\sigma}} \sigma_{v}=+0.005 \mathrm{eV}\left(\boldsymbol{\Delta}_{\boldsymbol{s} \boldsymbol{o}} \sigma_{v}=-0.006 \mathrm{eV}\right)$.

Chemical potential, $\mu$, is related to the charge transfer from a system to another having a lower value of $\mu$. Hence, it is anticipated that the odd-numbered $\mathrm{Au}_{\mathrm{n}}$ clusters present higher $\mu$ values because they have an open shell and that after transferring one electron, they will close their electronic shell and will be more stable than their original openshell clusters. The variation of chemical potential with and without considering spin-orbit coupling (SOC) effect is depicted in Figure 3c. According to this Figure, the vertical chemical potential of obtuse $\mathrm{Au}_{3}$ has the highest value, with or without considering spin-orbit coupling $\left(\mu_{\mathrm{v}}=-4.712 \mathrm{eV}, \mu_{\mathrm{v}, \mathrm{so}}=-4.750 \mathrm{eV}\right)$, indicating its high spontaneous response and chemical reactivity.

Figure $4 \mathrm{c}$ shows the spin-orbit correction to the chemical potential of neutral gold clusters versus the cluster size. Spin-orbit coupling increases the vertical chemical potential of $\mathrm{Au}_{2}$ by $0.021 \mathrm{eV}$, while decreases that of $\mathrm{Au}_{3} \mathrm{and} \mathrm{Au}_{6}$ 
by 0.038 and $0.037 \mathrm{eV}$, respectively. Hence, spin-orbit coupling has more important effect on the chemical potential of $\mathrm{Au}_{3}$.

Electrophilicity has been a measure of the energy stabilization of a cluster due to acquiring additional electronic charge from its surroundings. It is expected that the electrophilicity index ( $\omega)$ should be related to the electron affinity (EA), because both $\omega$ and EA measure the capability of an agent to accept electrons. However, EA reflects the capability of accepting only one electron from the environment, whereas the electrophilicity index $(\omega)$ measures the energy lowering of a cluster due to maximal electron flow between donor and acceptor. The electron flows may be either less or more than one. Meanwhile, the electrophilicity index depends not only on EA, but also on IP. Electron affinity and electrophilicity index are related; yet they are not equal [20].

Table 2. Scalar-relativistic vertical ionization potential (vIP/eV), vertical electron affinity (vEA/eV), vertical chemical potential $\left(\mu_{\mathrm{v}} / \mathrm{eV}\right)$, vertical hardness $\left(\eta_{\mathrm{v}} / \mathrm{eV}\right)$, vertical softness $\left(\sigma_{\mathrm{v}} / \mathrm{eV}^{-1}\right)$ and vertical electrophilicity index $\left(\omega_{\mathrm{v}} / \mathrm{eV}\right)$

\begin{tabular}{|c|c|c|c|c|c|c|c|}
\hline $\mathbf{n}$ & & vIP/eV & vEA/eV & $\mu_{\mathrm{v}} / \mathrm{eV}$ & $\eta_{v} / e V$ & $\sigma_{\mathrm{v}} / \mathrm{eV}^{-1}$ & $\omega_{\mathrm{v}} / \mathrm{eV}$ \\
\hline 2 & & 9.529 & 2.201 & -5.865 & 3.664 & 0.273 & 4.694 \\
\hline 3 & (obtuse) & 7.561 & 1.863 & -4.712 & 2.849 & 0.351 & 3.897 \\
\hline 3 & (acute) & 7.561 & 1.861 & -4.711 & 2.850 & 0.351 & 3.894 \\
\hline 4 & & 8.279 & 2.840 & -5.560 & 2.720 & 0.368 & 5.683 \\
\hline 5 & & 8.082 & 3.364 & -5.723 & 2.359 & 0.424 & 6.942 \\
\hline 6 & & 8.234 & 2.480 & -5.357 & 2.877 & 0.348 & 4.987 \\
\hline
\end{tabular}

Subscript ' $v$ ' indicates 'vertical' properties.

Table 3. Spin-obit corrected vertical ionization potential $\left(\mathrm{vIP}_{\mathrm{so}} / \mathrm{eV}\right)$, vertical electron affinity $\left(\mathrm{vEA} \mathrm{Aso}_{\mathrm{s}} / \mathrm{eV}\right)$, vertical chemical potential $\left(\mu_{\mathrm{v}, \mathrm{s}_{0}} / \mathrm{eV}\right)$, vertical hardness $\left(\eta_{\mathrm{v}, \mathrm{s}_{0}} / \mathrm{eV}\right)$, vertical softness $\left(\sigma_{\mathrm{v}, \mathrm{s}_{0}} / \mathrm{eV}^{-1}\right)$ and vertical electrophilicity index $\left(\omega_{\mathrm{v}, s_{0}} / \mathrm{eV}\right)$

\begin{tabular}{|c|c|c|c|c|c|c|c|}
\hline $\mathbf{n}$ & & $\mathbf{v I P}_{\mathrm{so}} / \mathrm{eV}$ & $\mathbf{v E A}_{\mathrm{so}} / \mathrm{eV}$ & $\mu_{\mathrm{v}, \mathrm{so}} / \mathrm{eV}$ & $\eta_{\mathbf{v}, \mathbf{s o}_{0}} / \mathrm{eV}$ & $\sigma_{\mathrm{v}, \mathrm{so}} / \mathrm{e} \mathbf{V}^{-1}$ & $\omega_{\mathrm{v}, \mathrm{so}_{0}} / \mathrm{eV}$ \\
\hline 2 & & 9.489 & 2.199 & -5.844 & 3.645 & 0.274 & 4.685 \\
\hline 3 & (obtuse) & 7.561 & 1.938 & -4.750 & 2.812 & 0.356 & 4.012 \\
\hline 3 & (acute) & 7.561 & 1.937 & -4.749 & 2.812 & 0.356 & 4.010 \\
\hline 4 & & 8.286 & 2.837 & -5.562 & 2.725 & 0.367 & 5.676 \\
\hline 5 & & 8.079 & 3.368 & -5.724 & 2.356 & 0.424 & 6.953 \\
\hline 6 & & 8.316 & 2.472 & -5.394 & 2.922 & 0.342 & 4.979 \\
\hline
\end{tabular}

Subscripts ' $v$ ' and 'so' indicate 'vertical properties' and 'spin-orbit', respectively.

Table 4. Scalar-relativistic adiabatic ionization potential (aIP/eV), adiabatic electron affinity (aEA/eV), adiabatic chemical potential $\left(\mu_{\mathrm{a}} / \mathrm{eV}\right)$, adiabatic hardness $\left(\eta_{\mathrm{a}} / \mathrm{eV}\right)$, adiabatic softness $\left(\sigma_{\mathrm{a}} / \mathrm{eV}^{-1}\right)$ and adiabatic electrophilicity index $\left(\omega_{\mathrm{a}} / \mathrm{eV}\right)$

\begin{tabular}{cccccccc}
\hline $\mathbf{n}$ & & $\mathbf{a I P} / \mathbf{e V}$ & $\mathbf{a E A} / \mathbf{e V}$ & $\boldsymbol{\mu}_{\mathbf{a}} / \mathbf{e V}$ & $\boldsymbol{\eta}_{\mathbf{a}} / \mathbf{e V}$ & $\boldsymbol{\sigma}_{\mathbf{a}} / \mathbf{e V}^{-1}$ & $\boldsymbol{\omega}_{\mathbf{a}} / \mathbf{e V}$ \\
\hline 2 & & 9.523 & 2.221 & -5.872 & 3.651 & 0.274 & 4.722 \\
3 & (obtuse) & 7.547 & 3.145 & -5.346 & 2.201 & 0.454 & 6.492 \\
3 & (acute) & 7.547 & 2.199 & -4.873 & 2.674 & 0.374 & 4.440 \\
4 & & 8.240 & 2.878 & -5.559 & 2.681 & 0.373 & 5.763 \\
5 & & 8.009 & 3.381 & -5.695 & 2.314 & 0.432 & 7.008 \\
6 & & 8.182 & 2.528 & -5.355 & 2.827 & 0.354 & 5.072 \\
\hline
\end{tabular}

Subscript 'a' indicates 'adiabatic' properties.

Table 5. Spin-obit corrected adiabatic ionization potential $\left(\mathrm{aIP}_{\mathrm{so}} / \mathrm{eV}\right)$, adiabatic electron affinity $\left(\mathrm{aEA} \mathrm{A}_{\mathrm{so}} / \mathrm{eV}\right)$, adiabatic chemical

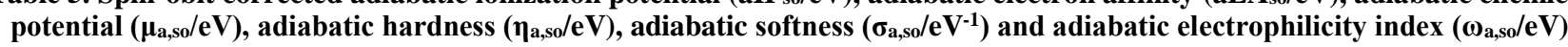

\begin{tabular}{|c|c|c|c|c|c|c|c|}
\hline $\mathbf{n}$ & & $\mathrm{aIP}_{\mathrm{so}} / \mathrm{eV}$ & $\mathrm{aEA}_{\mathrm{so}} / \mathrm{eV}$ & $\mu_{\mathrm{a}, \mathrm{s}_{0}} / \mathrm{eV}$ & $\eta_{\mathrm{a}, \mathrm{ss}_{0}} / \mathrm{eV}$ & $\sigma_{\mathrm{a}, \mathrm{so}_{\mathrm{o}}} / \mathrm{eV}^{-1}$ & $\omega_{\mathrm{a}, \mathrm{s}_{\mathrm{o}}} / \mathrm{eV}$ \\
\hline 2 & & 9.482 & 2.218 & -5.850 & 3.632 & 0.275 & 4.711 \\
\hline 3 & (obtuse) & 7.547 & 3.162 & -5.355 & 2.193 & 0.456 & 6.538 \\
\hline 3 & (acute) & 7.547 & 2.231 & -4.889 & 2.658 & 0.376 & 4.496 \\
\hline 4 & & 8.248 & 2.875 & -5.562 & 2.687 & 0.372 & 5.757 \\
\hline 5 & & 8.009 & 3.386 & -5.698 & 2.312 & 0.433 & 7.021 \\
\hline 6 & & 8.199 & 2.514 & -5.357 & 2.843 & 0.352 & 5.047 \\
\hline
\end{tabular}

Subscripts 'a' and 'so' indicate 'adiabatic properties' and 'spin-orbit', respectively. 
Table 6. Spin-orbit coupling (SOC) corrections to the vertical and adiabatic chemical potential $\left(\Delta_{s o} \mu / \mathrm{eV}\right)$, hardness $\left(\Delta_{s o} \eta / \mathrm{eV}\right)$, softness $\left(\Delta_{s o} \sigma / \mathrm{eV}^{-1}\right)$ and electrophilicity index $\left(\Delta_{s o} \omega / \mathrm{eV}\right)$

\begin{tabular}{|c|c|c|c|c|c|c|c|c|c|}
\hline n & & $\Delta_{s o} \mu_{v} / \mathrm{eV}$ & $\Delta_{s o} \mu_{a} / \mathrm{eV}$ & $\Delta_{s o} \eta_{v} / \mathrm{eV}$ & $\Delta_{s o} \eta_{a} / \mathrm{eV}$ & $\Delta_{s o} \sigma_{v} / \mathrm{eV}^{-1}$ & $\Delta_{s o} \sigma_{a} / \mathrm{eV}^{-1}$ & $\Delta_{s o} \omega_{v} / \mathrm{eV}$ & $\Delta_{s o} \omega_{a} / \mathrm{eV}$ \\
\hline 2 & & 0.021 & 0.022 & -0.019 & -0.019 & 0.001 & 0.001 & -0.009 & -0.011 \\
\hline 3 & (obtuse) & -0.038 & -0.009 & -0.037 & -0.008 & 0.005 & 0.002 & 0.115 & 0.046 \\
\hline 3 & (acute) & -0.038 & -0.016 & -0.038 & -0.016 & 0.005 & 0.002 & 0.116 & 0.056 \\
\hline 4 & & -0.002 & -0.003 & 0.005 & 0.006 & -0.001 & -0.001 & -0.007 & -0.006 \\
\hline 5 & & -0.001 & -0.003 & -0.003 & -0.002 & 0.000 & 0.001 & 0.011 & 0.013 \\
\hline 6 & & -0.037 & -0.002 & 0.045 & 0.016 & -0.006 & -0.002 & -0.008 & -0.025 \\
\hline
\end{tabular}

Subscripts 'v', 'a' and 'so' indicate 'vertical', 'adiabatic' and 'spin-orbit', respectively. Obtuse and acute stand for the two Jahn-Teller components of gold trimer.
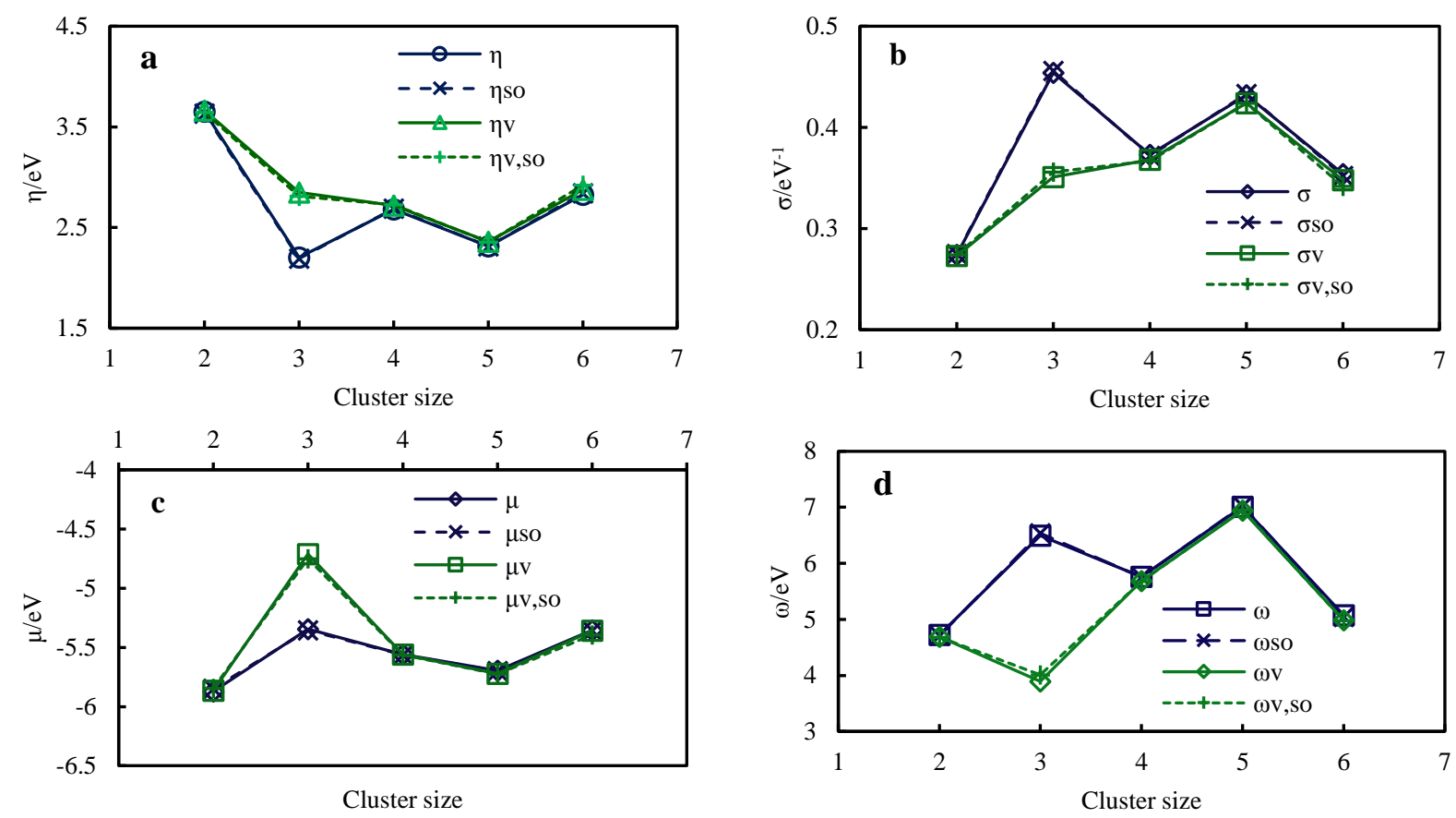

Figure 3. Variation of the (a) chemical hardness, (b) softness, (c) chemical potential and (d) electrophilicity index values with cluster size. Subscripts 'so' and ' $v$ ' indicate 'spin-orbit' and 'vertical', respectively

The variation of electrophilicity index as a function of cluster size, with and without considering spin-orbit coupling (SOC) effect is plotted in Figure 3d. As can be seen in this Figure, the electrophilicity index computed using adiabatic electron affinities and ionization potentials shows an odd-even oscillation behavior. The odd-numbered gold clusters present local maxima, and due to their open-shells, have more tendencies to accept electronic charge. On the other hand, the even-numbered and closed shell gold clusters are more stable and are less likely to acquire additional electronic charge. Hence, low electrophilicity index values are expected for them. However, using vertical IPs and EAs, the electrophilicity index behaves similar to vertical electron affinity (Table 2), and due to the linear geometry of the stable anionic gold trimer [33], acute $\mathrm{Au}_{3}$ has a very low vertical electrophilicity index $\left(\omega_{\mathrm{v}}=3.894\right.$ and $\omega_{\mathrm{v}, \mathrm{s}}=4.010$ $\mathrm{eV}$ ) and the even-odd alternation is not observed (Figure 3d). To illustrate the spin-orbit coupling effect on the electrophilicity index of small neutral gold clusters, the variation of spin-orbit correction to the $\omega$ as a function of cluster size is presented in Figure 4d. An odd-even alternation behavior is obvious. Moreover, the spin-orbit correction to the vertical and adiabatic electrophilicity values of acute $\mathrm{Au}_{3}\left(\Delta_{s o} \omega_{v}=0.116 \mathrm{eV}\right.$ and $\left.\Delta_{s o} \omega_{a}=0.056 \mathrm{eV}\right)$ and $\mathrm{Au}_{5}$ $\left(\Delta_{s o} \omega_{v}=0.011 \mathrm{eV}\right.$ and $\Delta_{s o} \omega_{a}=0.013 \mathrm{eV}$ ) are positive, indicating that spin-orbit coupling increases the electrophilicity index of these clusters. It is evident that spin-orbit coupling has significant effect on the vertical electrophilicity index of the gold trimer.

Maximal charge acceptance, $\Delta N_{\max }=-\mu / \eta$, measures the maximal flow of electrons between a donor and an acceptor [20]. Figure 5a depicts the variation of the maximal charge acceptance of neutral gold clusters as a function of cluster size, with and without considering spin-orbit coupling. Similar to the electrophilicity index (Figure 3d), the adiabatic maximal charge acceptance exhibits odd-even alternation behavior, whether spin-orbit coupling is considered or not. Furthermore, the open-shell and odd-numbered $\mathrm{Au}_{3}$ and $\mathrm{Au}_{5}$ clusters have high adiabatic maximal charge acceptance values of 2.442 and 2.465 , respectively, i.e. these clusters show high tendency to accept electrons. 
The spin-orbit coupling corrected adiabatic maximal charge acceptance per atom of neutral gold clusters are 0.805 , 0.814 (0.613), 0.517, 0.493 and 0.314 atom $^{-1}$ for $\mathrm{Au}_{2}$, obtuse (acute) $\mathrm{Au}_{3}, \mathrm{Au}_{4}, \mathrm{Au}_{5}$ and $\mathrm{Au}_{6}$, respectively. Obtuse $\mathrm{Au}_{3}$ has the highest adiabatic maximal charge acceptance per atom value of 0.814 and 0.810 atom $^{-1}$ with and without considering spin-orbit coupling, respectively; indicating high reactivity of this cluster due to its active sites. On the other hand, $\mathrm{Au}_{6}$ has the lowest adiabatic maximal charge acceptance per atom value of 0.314 and 0.316 atom ${ }^{-1}$ with and without considering spin-orbit coupling, respectively, indicating its high stability. The results of Nguyen et al. [12] based on the second difference of energy and fragmentation energy calculations, also predicted high reactivity (stability) for $\mathrm{Au}_{3}\left(\mathrm{Au}_{6}\right)$. As can be seen in Figure $5 \mathrm{~b}$, the spin-orbit correction to the maximal charge acceptance of neutral gold clusters exhibits an odd-even alternation behavior. Moreover, when the maximal charge acceptance is computed using vertical ionization potentials and electron affinities, inclusion of spin-orbit coupling increases the maximal charge acceptance of obtuse $\mathrm{Au}_{3}$ more $(0.035 \mathrm{eV})$ and thus destabilizes it more. The natural population analysis (NPA) shows that the natural charge is positive (negative) for the apex atom of acute (obtuse) gold trimer, i.e. the natural charge changes its sign in going from acute $\left({ }^{2} A_{1}\right)$ to the obtuse $\left({ }^{2} B_{1}\right) A_{3}$ [35-38]. Figure 6 represents the natural charges and electrostatic potential (ESP) contour maps of the lowest-energy gold clusters $\mathrm{Au}_{\mathrm{n}}(\mathrm{n}-3-6)$, at the DKH2-B3P86/DZP-DKH level. It is obvious that the apex atom of acute gold trimer has the highest natural charge value of +0.172 , indicating its capability to accept additional electronic charge. Red lines in the electrostatic potential contour maps correspond to the negative charges, so these regions are responsible for the nucleophilic interactions.
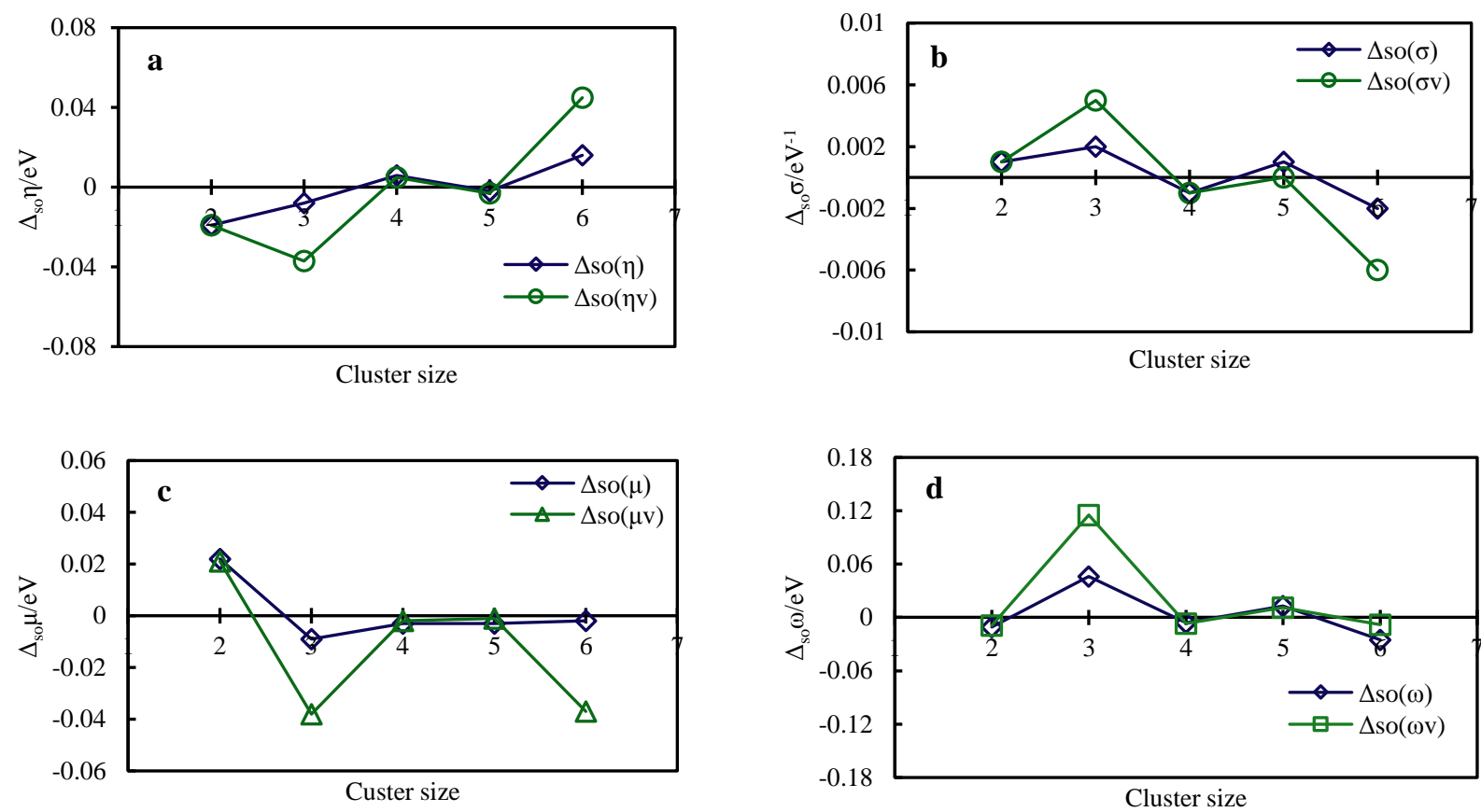

Figure 4. Spin-orbit corrections to the (a) chemical hardness, (b) softness, (c) chemical potential and (d) electrophilicity index. Subscripts 'so' and ' $v$ ' indicate 'spin-orbit' and 'vertical', respectively
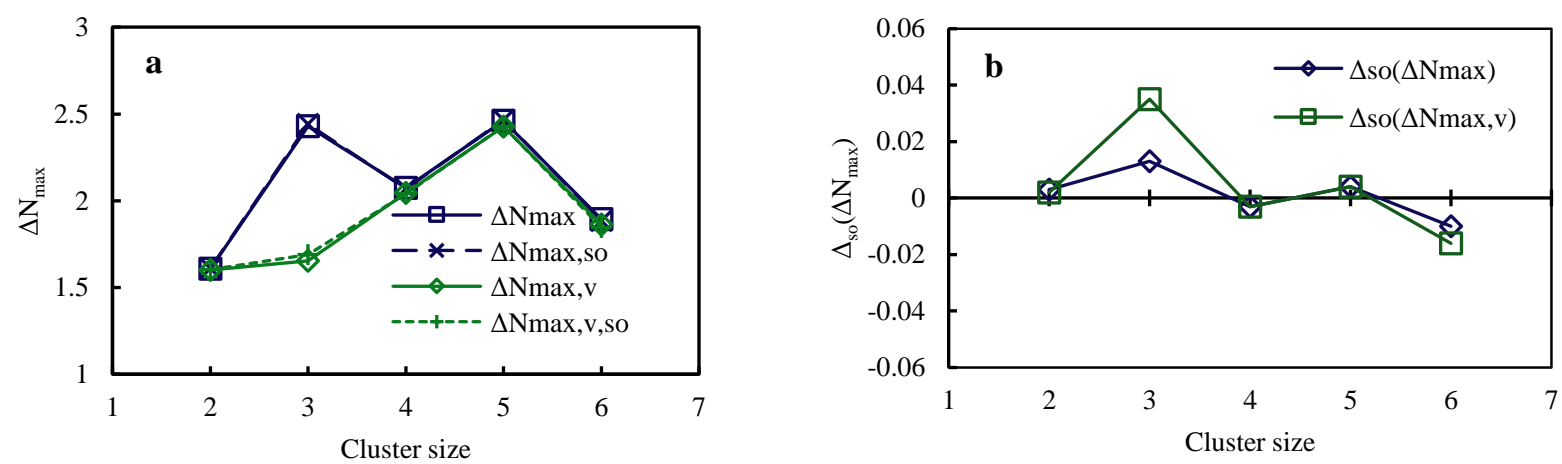

Figure 5. Variation of (a) maximal charge acceptances with and without considering spin-orbit coupling, (b) spin-orbit corrections to the maximal charge acceptance. Subscripts 'so' and ' $v$ ' indicate 'spin-orbit' and 'vertical', respectively 


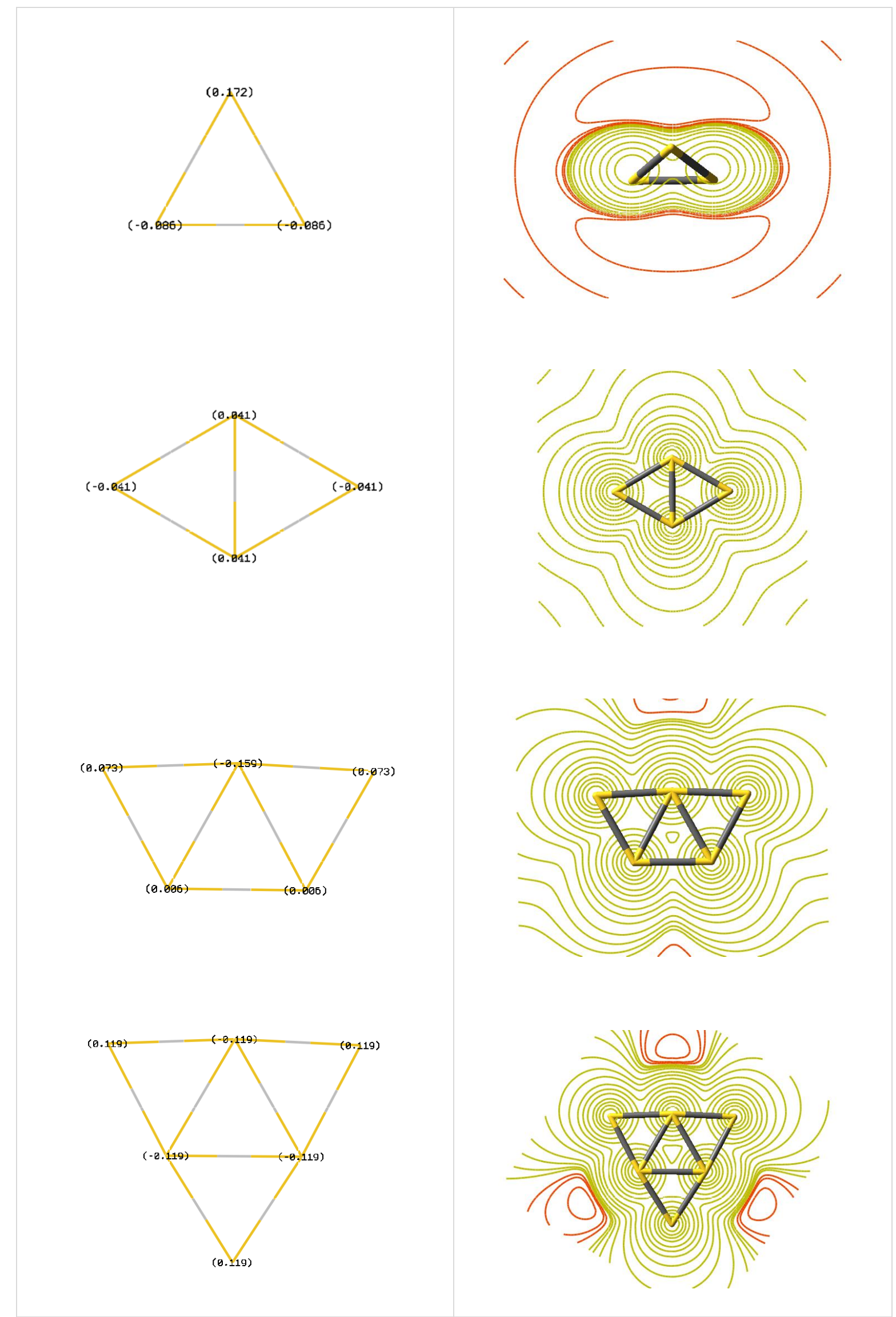

Figure 6. Electrostatic potential (ESP) contour maps (right panel) and natural charge values (left panel) of neutral gold clusters $\mathrm{Au}_{\mathrm{n}}(\mathrm{n}=3-6)$ 


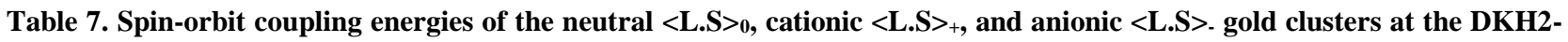
B3P86/DZP-DKH//DKHSO-GHF/DZP-DKH level. The SOC energies of the cationic and anionic clusters are at the optimized geometries of neutral clusters.

\begin{tabular}{|c|c|c|c|c|c|c|}
\hline $\mathbf{n}$ & $\langle\text { L.S }\rangle_{0}$ (a.u.) & $\langle\text { L.S }\rangle_{+}$(a.u.) & $\langle$L.S $\rangle$. (a.u.) & $\left\langle\mathbf{L} . \mathrm{S}>_{0} / \mathbf{n}(\right.$ a.u./atom) & $\langle\mathbf{L} . \mathbf{S}\rangle_{+} / \mathbf{n}($ a.u./atom) & $<$ L.S $>$./n (a.u./atom) \\
\hline 2 & -0.15304 & -0.15451 & -0.15298 & -0.07652 & -0.07726 & -0.07649 \\
\hline 3 (obtuse) & -0.22862 & -0.22946 & -0.23214 & -0.07621 & -0.07649 & -0.07738 \\
\hline 3 (acute) & -0.22943 & -0.22945 & -0.23219 & -0.07648 & -0.07648 & -0.07740 \\
\hline 4 & -0.30670 & -0.30641 & -0.30659 & -0.07668 & -0.07660 & -0.07665 \\
\hline 5 & -0.38385 & -0.38395 & -0.38400 & -0.07677 & -0.07679 & -0.07680 \\
\hline 6 & -0.46151 & -0.45848 & -0.46120 & -0.07692 & -0.07641 & -0.07687 \\
\hline
\end{tabular}

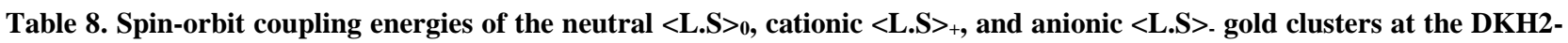
B3P86/DZP-DKH//DKHSO-GHF/DZP-DKH level. $\langle\text { L.S }\rangle_{0},\langle\text { L.S }\rangle_{+}$, and $\langle\text {L.S }\rangle_{\text {. are }}$ the SOC energies at the optimized geometries of neutral, cationic and anionic gold clusters, respectively.

\begin{tabular}{|c|c|c|c|c|c|c|}
\hline $\mathbf{n}$ & $\langle\text { L.S }\rangle_{0}$ (a.u.) & $\langle\text { L.S }\rangle_{+}$(a.u.) & $\langle$L.S $\rangle$. (a.u.) & $\left\langle\right.$L.S $>_{0} / \mathbf{n}$ (a.u./atom) & $\langle\text { L.S }\rangle_{+} /$n (a.u./atom) & $<$L.S $>$/n (a.u./atom) \\
\hline 2 & -0.15304 & -0.15455 & -0.15293 & -0.07652 & -0.07728 & -0.07647 \\
\hline 3 (obtuse) & -0.22862 & -0.22945 & -0.23006 & -0.07621 & -0.07648 & -0.07669 \\
\hline 3 (acute) & -0.22943 & -0.22945 & -0.23060 & -0.07648 & -0.07648 & -0.07687 \\
\hline 4 & -0.30670 & -0.30643 & -0.30661 & -0.07668 & -0.07661 & -0.07665 \\
\hline 5 & -0.38385 & -0.38386 & -0.38403 & -0.07677 & -0.07677 & -0.07681 \\
\hline 6 & -0.46151 & -0.46085 & -0.46101 & -0.07692 & -0.07681 & -0.07683 \\
\hline
\end{tabular}
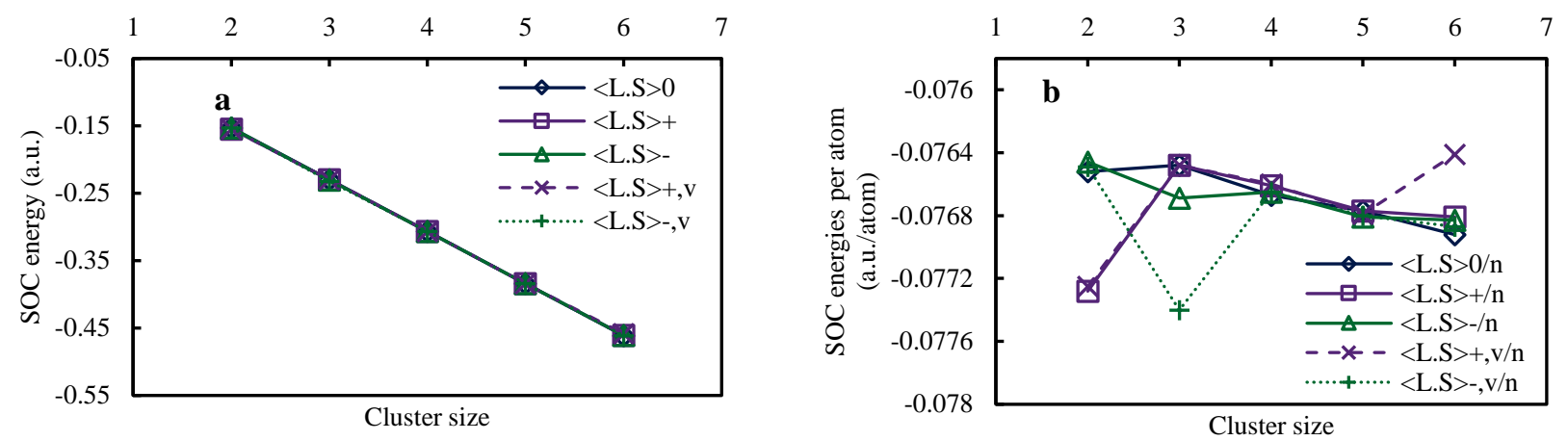

Figure 7. Variation of (a) Maximal Spin-orbit coupling energies of the neutral $\left.\left(<L_{. S}\right\rangle_{0}\right)$, cationic $\left.(<\text { L.S }\rangle_{+}\right)$and anionic $\left.(<\text { L.S }\rangle_{-}\right)$gold clusters, (b) Spin-orbit coupling energies per atom of the neutral $\left.(<\text {L.S }\rangle_{0} / \mathbf{n}\right)$, cationic $\left(\left\langle L_{. S} \text { S }\right\rangle_{+} / \mathbf{n}\right)$ and anionic $(<$ L.S $>$./n) gold clusters, at the DKH2-B3P86/DZP-DKH//DKHSO-GHF/DZP-DKH level of theory. Subscript ' $v$ ' indicates 'vertical'.

According to Equations 3 to 6, the chemical potential, hardness, softness and electrophilicity index are computed based on ionization potentials IP $=\mathrm{E}\left[\mathrm{Au}_{\mathrm{n}}{ }^{+}\right]-\mathrm{E}\left[\mathrm{Au}_{\mathrm{n}}\right]$ and electron affinities $\mathrm{EA}=\mathrm{E}\left[\mathrm{Au}_{\mathrm{n}}\right]-\mathrm{E}\left[\mathrm{Au}_{\mathrm{n}}{ }^{-}\right]$, where $\mathrm{E}\left[\mathrm{Au}_{\mathrm{n}}\right]$, $\mathrm{E}\left[\mathrm{Au}_{\mathrm{n}}{ }^{+}\right]$and $\mathrm{E}\left[\mathrm{Au}_{\mathrm{n}}{ }^{-}\right]$are the total energies of the neutral, cationic and anionic clusters, respectively. As a result, based on Equation 1, the spin-orbit corrections to the chemical potential, hardness, softness and electrophilicity index depend on the spin-orbit energies of the neutral, positively and negatively charged gold clusters, i.e. $\langle\text { L. } S\rangle_{0},\langle L . S\rangle_{+}$and $\langle L . S\rangle_{-}$. In the following, the behavior of spin-orbit coupling energy $\Delta_{S O} E=\langle L . S\rangle$ (Equation 1) is investigated. Tables 7 and 8 present the calculated spin-orbit coupling (SOC) energies for the neutral, cationic and anionic gold clusters. Figure 7a depicts the variation of the SOC energies versus the cluster size. From this Figure, a linear relationship is observed between the spin-orbit coupling energies and the cluster size. Furthermore, the values of SOC energies, $\langle L . S\rangle_{0},\langle L . S\rangle_{+}$and $\langle L . S\rangle_{-}$, vary in a relatively broad range, i.e. between -0.152 and -0.462 au. According to Tables 7 and 8, the spin-orbit coupling (SOC) energies of neutral open-shell and odd-numbered $\mathrm{Au}_{3}$ and $\mathrm{Au}_{5}$ with one unpaired electron are larger than those of positively and negatively charged closed shell gold trimer and pentamer, i.e. $\langle L . S\rangle_{0}$ is larger than $\langle L . S\rangle_{+}$and $\langle L . S\rangle_{-}$. Hence, unpaired electron(s) can result in higher SOC energies. Moreover, the geometry of a cluster may influence the $\Delta_{S O} E=\langle L . S\rangle$ magnitude due to appropriate orientations of the total orbital and spin angular momentum vectors. The variation of spin-orbit coupling energies per atom, i.e. $\langle L . S\rangle_{0} / n,\langle L . S\rangle_{+} / n$ and $\langle L . S\rangle_{-} / n$ versus the cluster size is plotted in Figure $7 \mathrm{~b}$. From this Figure, considerable separations among the SOC energies per atom of the neutral, positively and negatively charged $\mathrm{Au}_{2}, \mathrm{Au}_{3}$, and $\mathrm{Au}_{6}$ clusters are observed. Furthermore, the separations of the spin-orbit coupling (SOC) energies per atom of the gold trimer are larger, resulting in higher SOC effects on the electronic properties of this cluster. 


\section{Conclusion}

Due to their biocompatibility and unique physical properties, gold clusters are attracting interest as the building blocks of novel nano-structural materials. Most of the computations on small neutral gold clusters have been performed using scalar-relativistic methods. However, the Spin-Orbit Coupling (SOC) effect appears to be important. Previous studies focused on spin-orbit coupling effects on HOMO-LUMO energy gaps, geometries, cohesive energies, and optical absorption of gold clusters. The electrophilicity index is related to the energy level associated with the maximum amount of electron flow between a donor and an acceptor and provides information about structure, stability, reactivity, and interactions. In the present research work, the behaviour of the electrophilicity index along with chemical potential, hardness, and softness is investigated. The main purpose is to examine spin-orbit coupling (SOC) effects on these properties. We use generalized Hartree-Fock method in conjunction with the fourth-order Douglas-Kroll-Hess (DKH) Hamiltonian and all-electron relativistic double- $\zeta$-level basis set to compute the spin orbit coupling energies and corrections to the electrophilicity index, chemical potential, hardness and softness. It is revealed that spin-orbit correction to the vertical chemical hardness has an important effect on $\mathrm{Au}_{3}$ and $\mathrm{Au}_{6}$ by decreasing (increasing) the hardness of the gold trimer (hexamer). Moreover, spin-orbit correction to the softness exhibits an even-odd oscillation behavior, and the inclusion of SOC increases (decreases) the softness of $\mathrm{Au}_{3}\left(\mathrm{Au}_{6}\right)$ and thus destabilizes (stabilizes) it. Spin-orbit coupling has a more important effect on the chemical potential of the gold trimer by decreasing its value. The adiabatic electrophilicity index shows an odd-even alternation behaviour and oddnumbered gold clusters present local maxima due to their open-shells. The spin-orbit corrections to the electrophilicity index of acute $\mathrm{Au}_{3}$ and $\mathrm{Au}_{5}$ are positive, i.e., SO coupling increases the electrophilicity index of these clusters. Furthermore, the open-shell and odd-numbered gold trimers have the highest adiabatic maximal charge acceptance per atom, with and without considering spin-orbit coupling, indicating the high reactivity of this cluster. In addition, spinorbit coupling increases the maximal charge acceptance of $\mathrm{Au}_{3}$ more and thus destabilizes it more. The natural population analysis reveals that the apex atom of the acute gold trimer has the highest natural charge, indicating its capability to accept additional electronic charges.

\section{Supplementary Material}

Supplementary material (Appendix I) for this article contains XYZ coordinates of the lowest-energy gold clusters optimized at the DKH2-B3P86/DZP-DKH level of theory.

\section{Declaration of Competing Interest}

The authors declare that they have no known competing financial interests or personal relationships that could have appeared to influence the work reported in this paper.

\section{References}

[1] Liu, W., Zhang, Z., Zhang, Z. M., Hao, P., Ding, K. \& Li, Z. (2019). Integrated phenotypic screening and activity-based protein profiling to reveal potential therapy targets of pancreatic cancer. Chem. Commun., 55, 1596-1599. doi:10.1039/C8CC08753A.

[2] Kurdekar, A. D., Chunduri, L. A. A., Manohar, C. S., Haleyurgirisetty, M. K., Hewlett, I. K. \& Venkataramaniah, K. (2018). Streptavidin-conjugated gold nanoclusters as ultrasensitive fluorescent sensors for early diagnosis of HIV infection. Sci. Adv., 4, eaar6280. doi:10.1126/sciadv.aar6280.

[3] Kaur, N., Aditya, R. N., Singh, A. \& Kuo, T. R. (2018). Biomedical Applications for Gold Nanoclusters: Recent Developments and Future Perspectives. Nanoscale Res. Lett., 13, 302. doi:10.1186/s11671-018-2725-9.

[4] Liu, M., Tang, F., Yang, Z., Z. Xu, J. \& Yang, X. (2019). Recent Progress on Gold-Nanocluster-Based Fluorescent Probe for Environmental Analysis and Biological Sensing. J. Anal. Methods Chem., 2019, 1-10. doi:10.1155/2019/1095148.

[5] Xu, D. D., Zheng, B., Song, C. Y., Lin, Y., Pang, D. W. \& Tang, H. W. (2019). Metal-enhanced fluorescence of gold nanoclusters as a sensing platform for multi-component detection. Sens. Actuators B Chem., 282, 650-658. doi:10.1016/j.snb.2018.11.122.

[6] Liu, L. \& Corma, A. (2018). Metal Catalysts for Heterogeneous Catalysis: From Single Atoms to Nanoclusters and Nanoparticles. Chem. Rev., 118, 4981-5079. doi:10.1021/acs.chemrev.7b00776.

[7] Higaki, T., Li, Y., Zhao, S., Li, Q., Li, S., Du, X. S., Yang, S., Chai, J. \& Jin, R. (2019). Atomically Tailored Gold Nanoclusters for Catalytic Application. Angew. Chem. Int. Ed., 58, 8291-8302. doi:10.1002/anie.201814156.

[8] Kumar, B., Kawawaki, T., Shimizu, N., Imai, Y., Suzuki, D., Hossain, S., Nair, L. V. \& Negishi, Y. (2020). Gold nanoclusters as electrocatalysts: size, ligands, heteroatom doping, and charge dependences. Nanoscale, 12, 9969-9979. doi:10.1039/D0NR00702A.

[9] Jorge, F. E. \& Santos, A. S. (2018). Structures Stabilities Reactivities and (Hyper) Polarizabilities of Small Gold Clusters. J. Braz. Chem. Soc., 29, 838-844. doi:10.21577/0103-5053.20170207. 
[10] Baek, H., Moon, J. \& Kim, J. (2017). Benchmark Study of Density Functional Theory for Neutral Gold Clusters, Aun (n = 28). J. Phys. Chem. A, 121, 2410-2419. doi:10.1021/acs.jpca.6b11868.

[11] Singh, N. B. \& Sarkar, U. (2015). Geometry chemical reactivity and Raman spectra of gold clusters. Cogent Chem., 1, 1076713. doi:10.1080/23312009.2015.1076713.

[12] Nhat, P. V., Si, N. T., Leszczynski, J. \& Nguyen, M. T. (2017). Another look at structure of gold clusters Aun from perspective of phenomenological shell model. Chem. Phys., 493, 140-148. doi:10.1016/j.chemphys.2017.06.009.

[13] Xiao, L. \& Wang, L. (2004). From planar to three-dimensional structural transition in gold clusters and the spin-orbit coupling effect. Chem. Phys. Lett., 392, 452-455. doi:10.1016/j.cplett.2004.05.095.

[14] Shi, Y. K., Li, Z. H. \& Fan, K. N. (2010). Validation of Density Functional Methods for the Calculation of Small Gold Clusters. J. Phys. Chem. A, 114, 10297-10308. doi:10.1021/jp105428b.

[15] Flores, M. A. \& Menéndez-Proupin, E. (2016). Spin-orbit coupling effects in gold clusters: The case of Au13. J. Phys. Conf. Ser., 720, 012034. doi:10.1088/1742-6596/720/1/012034.

[16] Rusakov, A. A., Rykova, E., Scuseria, G. E. \& Zaitsevskii, A. (2007). Importance of spin-orbit effects on the isomerism profile of Au3: An ab initio study. J. Chem. Phys., 127, 164322. doi:10.1063/1.2795710.

[17] Afshar, M. \& Sargolzaei, M. (2013). Spin and orbital magnetism of coinage meta trimers (Cu3, Ag3, Au3): A relativistic density functional theory study. AIP Adv., 3, 112122. doi:10.1063/1.4834336.

[18] Shayeghi, A., Pašteka, L. F., Götz, D. A., Schwerdtfeger, P. \& Schäfer, R. (2018). Spin-orbit effects in optical spectra of goldsilver trimers. Phys. Chem. Chem. Phys., 20, 9108-9114. doi:10.1039/c8cp00672e.

[19] Jiang, D. E., Kühn, M., Tang, Q. \& Weigend, F. (2014). Superatomic Orbitals under Spin-Orbit Coupling. J. Phys. Chem. Lett., 5, 3286-3289. doi:10.1021/jz501745z.

[20] Parr, R. G., Szentpály, L. v., \& Liu, S. (1999). Electrophilicity Index. Journal of the American Chemical Society, 121(9), 1922-1924. doi:10.1021/ja983494x.

[21] Chattaraj, P. K., Sarkar, U. \& Roy, D. R. (2006). Electrophilicity Index. Chem. Rev., 106, 2065-2091. doi:10.1021/cr040109f.

[22] Frisch, M. J., Trucks, G. W., Schlegel, H. B., Scuseria, G. E., Robb, M. A., Cheeseman, J. R., ...., Cioslowski, J. \& Fox, D. J. (2013). Gaussian 09, Gaussian, Inc., Wallingford, Revisions A.02 (2009) and D.01 (2013). Oxfordshire, England.

[23] Dennington, R. D., Keith, T. A. \& Millam, J. M. (2000-2008). GaussView, Version 5.0.9.

[24] Neto, A. C. \& Jorge, F. E. (2013). All-electron double zeta basis sets for the most fifth-row atoms: Application in DFT spectroscopic constant calculations. Chem. Phys. Lett., 582, 158-162. doi:10.1016/j.cplett.2013.07.045.

[25] Douglas, M. \& Kroll, N. M. (1974). Quantum Electrodynamical Corrections to the Fine Structure of Helium. Ann. Phys., 82, 89-155. doi:10.1016/0003-4916(74)90333-9.

[26] Jansen, G., \& Hess, B. A. (1989). Revision of the Douglas-Kroll transformation. Physical Review A, 39(11), 6016-6017. doi:10.1103/physreva.39.6016.

[27] Nakajima, T. \& Hirao, K. (2012). The Douglas-Kroll-Hess Approach. Chem. Rev., 112, 385-402. doi:10.1021/cr200040s.

[28] Reiher, M. (2012) Relativistic Douglas-Kroll-Hess theory. WIREs Comput. Mol. Sci., 2, 139-149. doi:10.1002/wcms.67.

[29] Reiher, M. \& Wolf, A. (2015). Douglas-Kroll-Hess Theory, in: Reiher, M. \& Wolf, A., Relativistic Quantum Chemistry: The Fundamental Theory of Molecular Science, Second Ed., WILEY-VCH, Germany, 469-501. doi:10.1002/9783527667550.ch12.

[30] Gruene, P., Butschke, B., Lyon, J. T., Rayner, D. M., \& Fielicke, A. (2014). Far-IR Spectra of Small Neutral Gold Clusters in the Gas Phase. Zeitschrift für physikalische Chemie, 228(4-5), 337-350. doi:10.1515/zpch-2014-0480.

[31] Hammes-Schiffer, S. \& Andersen, H. C. (1993). The advantages of the general Hartree-Fock method for future computer simulation of materials. J. Chem. Phys., 99 (3), 1901-1913. doi:10.1063/1.465305.

[32] Pearson, R. G. (1988). Absolute Electronegativity and Hardness: Application to Inorganic Chemistry. Inorg. Chem., 27, 734740. doi:10.1021/ic00277a030.

[33] Wesendrup, R., Hunt, T. \& Schwerdtfeger, P. (2000). Relativistic coupled cluster calculations for neutral and singly charged Au3 Clusters. J. Chem. Phys., 112, 9356. doi:10.1063/1.481556.

[34] Jorge, F. E., Ferreira, I. B., Soprani, D. D. \& Gomes, T. (2016). Estimating the Impact of an All-Electron Basis Set and Scalar Relativistic Effects on the Structure, Stability, and Reactivity of Small Copper Clusters. J. Braz. Chem. Soc., 27, $127-135$. doi:10.5935/0103-5053.20150261.

[35] Glendening, E. D., Reed, A. E., Carpenter, J. E. \& Weinhold. F. (1998). NBO Version 3.1. TCI, University of Wisconsin, Madison, United States. 
[36] Carvalho, F. S. \& Braga, J. P. (2018). DFT Study of Small Gold Clusters, Aun $(2 \leqslant \mathrm{n} \leqslant 6)$ : Stabilityand Charge Distribution Using M08-SO Functional. Brazilian Journal of Physics, 48, 390-397. doi:10.1007/s13538-018-0577-5.

[37] Nhat, P. V., Si, N. T., Tram, N. T. T., Duong, L. V., \& Nguyen, M. T. (2020). Elucidating the binding mechanism of thione containing mercaptopurine and thioguanine drugs to small gold clusters. Journal of Computational Chemistry, 41(19), 17481758. doi:10.1002/jcc.26216.

[38] Yadav, J., \& Saini, S. (2020). Atop adsorption of oxygen on small sized gold clusters: Analysis of size and site reactivity from restructuring perspective. Computational and Theoretical Chemistry, 1191, 113014. doi:10.1016/j.comptc.2020.113014. 


\section{Appendix I}

XYZ coordinates of the small neutral gold clusters optimized at the DKH2-B3P86/DZP-DKH level of theory.

$\begin{array}{llr}\mathbf{A u}_{2} & & \\ 0.000000 & 0.000000 & 1.190360 \\ 0.000000 & 0.000000 & -1.190360\end{array}$

$\mathrm{Au}_{3}$ (acute)

$0.000000 \quad 1.238856-0.738055$

$0.000000-1.238856-0.738055$

$0.000000 \quad 0.000000 \quad 1.476111$

$\mathrm{Au}_{5}$

$\begin{array}{lll}-1.267923 & 1.352279 & 0.001733\end{array}$

$\begin{array}{llll}-2.496312 & -0.862669 & -0.039240\end{array}$

$\begin{array}{lll}1.268335 & 1.352251 & 0.002184\end{array}$

$\begin{array}{lll}1.0 .000125 & -0.978835 & 0.074794\end{array}$

$\begin{array}{llll}2.496024 & -0.863026 & -0.039470\end{array}$

\author{
$\mathrm{Au}_{3}$ (obtuse) \\ $\begin{array}{lll}0.000000 & 1.239142 & -0.738019\end{array}$ \\ $\begin{array}{llll}0.000000 & -1.239142 & -0.738019\end{array}$ \\ $\begin{array}{llll}0.000000 & 0.000000 & 1.476038\end{array}$
}

$\mathrm{Au}_{4}$

$\begin{array}{lll}0.000000 & 1.278972 & 0.000421\end{array}$

$\begin{array}{llll}2.193981 & 0.000000 & -0.000421\end{array}$

$\begin{array}{llll}0.000000 & -1.278972 & 0.000421\end{array}$

$\begin{array}{llll}-2.193981 & 0.000000 & -0.000421\end{array}$

$\mathrm{Au}_{6}$

$\begin{array}{lll}-0.732383 & -1.360849 & 0.120640\end{array}$

$\begin{array}{lll}1.365676 & 2.537432 & -0.115246\end{array}$

$\begin{array}{lll}1.514815 & -2.451354 & -0.118110\end{array}$

$\begin{array}{lll}-0.813203 & 1.315450 & 0.115580\end{array}$

$\begin{array}{lll}1.545674 & 0.046071 & 0.115273\end{array}$

$\begin{array}{llll}-2.880579 & -0.086751 & -0.118137\end{array}$ 